\title{
Tradeoffs in Cherenkov Detection for Positron Emission Tomography
}

\author{
Andrada Muntean, Francesco Gramuglia, Esteban Venialgo, Student Member, IEEE, Claudio Bruschini, Senior \\ Member, IEEE, and Edoardo Charbon, Fellow, IEEE
}

\begin{abstract}
The coincidence time resolution (CTR) of a time-offlight positron emission tomography (TOF-PET) scanner is an essential parameter, which determines signal-to-noise ratio (SNR) in image reconstruction algorithms. CTR has an important impact on scintillation-based detectors used in TOFPET. Cherenkov PET has emerged as a prompt-photon based radiation detector, which has more accurate light-photon emission statistics. However, light transport becomes a dominant degradation effect in the light-photon detection statistics, which is highly correlated to the crystal length and consequently to the gamma-photon detection efficiency.

This work focuses on finding the optimal design in terms of timing resolution and gamma-photon detection efficiency for Cherenkov detectors. Radiator design considerations such as size, materials and coatings, along with electronic jitter, and dark count rate (DCR), are included in a mathematical simulation that determines their effect on timing resolution. Currently, two different scintillators were simulated, namely $\mathrm{PbF}_{2}$ and $\mathrm{PbWO}_{4}$, with a maximum Cherenkov count per frame of 12 photons; an electronic jitter of $4.3 \mathrm{ps}$ to $42.5 \mathrm{ps}$ (standard deviation) was added. Simulation results confirm the high impact of jitter on the uncertainty of arrival times of Cherenkov photons.
\end{abstract}

Index Terms - Cherenkov, time resolution, gamma detection efficiency, DCR, electronics jitter, Geant4, TOF-PET.

\section{INTRODUCTION}

$I_{\text {times }}^{N}$ $\mathrm{N}$ recent years, considerable interest has been dedicated to time-of-flight PET (TOF-PET), especially for improving coincidence time resolution (CTR) [1]. Better CTR can lead to increased signal-to-noise ratio (SNR) in PET images and higher overall medical imaging quality [2].

CTR in scintillator based detectors has fundamental limitations [3]. Cherenkov luminescence has emerged as a prompt-photon generation mechanism to further improve timing performance. This effect arises from the interaction between a charged particle travelling faster than the phase velocity of light in a dielectric medium. In Cherenkov-based radiation detectors, timing resolution is highly correlated with photodetector response and light-transport time spread in the radiator, due to the low number of emitted photons and very fast generation time. Intuitively, by reducing the dimensions of the Cherenkov radiator and by suppressing reflections with absorbing coatings we can improve timing performance. However, this leads to a smaller number of Cherenkov photons reaching the detector, and subsequently to a lower

Andrada Muntean, Francesco Gramuglia, Claudio Bruschini and Edoardo Charbon are with EPFL, Lausanne, Switzerland. Esteban Venialgo is with Delft University of Technology, Delft, The Netherlands. The research has been supported, in part, by the Swiss National Science Foundation. gamma-photon detection efficiency. The number of detected Cherenkov photons is limited by the photon detection efficiency (PDE) of the photodetector, which is typically low near the ultraviolet spectrum [4] [5].

The current trend is the use of silicon photomultipliers (SiPMs) as detectors of choice for medical applications, such as TOF-PET, due to their robustness, low bias voltages and tolerance to magnetic fields [4]. The detector non-idealities include single-photon timing resolution (SPTR), crosstalk, afterpulsing, and dark count rate (DCR). Fig. 1 represents a conceptual tradeoff diagram that takes into account all the aforementioned parameters that influence Cherenkov detection efficiency and timing.

This paper explores these tradeoffs by analyzing different crystal configurations (length, coatings and materials) and readout circuit effects, in particular total jitter, PDE, DCR, and crosstalk through the use of mathematical models. The overall goal is to determine an optimal detector design suitable for Cherenkov TOF-PET.

\section{Methodology}

The physics behind the time and spatial distribution of photons at the exit surface of the radiator is one of the most important aspects which needs to be considered. As a result, extensive simulations have been carried out in order to study the tradeoffs. Firstly, a simulation setup was implemented in the Geant4 toolkit [6], which represents a realistic case where gamma photons interact in a Cherenkov radiator. The simulations were performed using absorbing and reflective coating surfaces and their effect on the photon distribution at the detector was investigated. Two different radiators were considered, i.e. $(3 \times 3 \times 15) \mathrm{mm}^{3} \mathrm{PbWO}_{4}$ and $\mathrm{PbF}_{2}$. For future analysis, additional dimensions and types will be included and compared to the literature. A "classic" radiator was used as a reference for comparing the obtained results with what was reported in [7]. The key parameters of the simulated crystals

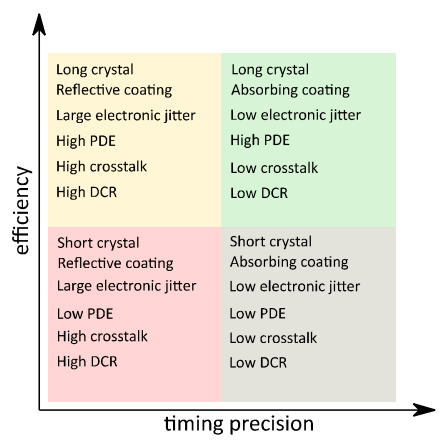

Fig. 1. Tradeoff diagram of Cherenkov detection efficiency. 
are presented in Table 1.

The resulting photon arrival time probability density function (PDF) extracted from the Geant4 simulations (Fig. 2) is convolved with a Gaussian distribution with a standard deviation of 4.3ps (10ps FWHM) to 42.5ps (100ps FWHM) that models the total instrumentation chain jitter. The lower limit of 10ps FWHM represents the desired goal for Cherenkov detection, while the upper limit represents the current electronic performance status.

Additional noise sources, such as afterpulsing and crosstalk, are not considered in these simulations, since in digital SiPMs (D-SiPMs) they can be reduced by utilizing active recharge and quenching techniques with an adjustable dead time [7].

\section{PRELIMINARY RESULTS}

Initial simulation results of $\mathrm{PbF}_{2}$ crystal for $4.3 \mathrm{ps}$ up to $42.5 \mathrm{ps}$ standard deviation jitter and no DCR are depicted in Fig. 3 as standard deviations of estimated arrival times for up to the first 12 detected Cherenkov photons. The average number of Cherenkov events per frame is 2, 6 and 12. The results confirm the significant influence of jitter on the timing estimation of Cherenkov photons as well as the degradation in precision due to the very small number of photon counts in a measurement frame. The same behavior is depicted in Fig. 4, where the standard deviation of a specific order is plotted as a function of jitter.

TABLE I

CRYSTAL PARAMETERS

\begin{tabular}{|c|c|c|c|}
\hline Parameter & $\mathrm{PbF}_{2}$ & $\mathrm{PWO}_{4}$ & BGO \\
\hline Refractive index ${ }^{\mathrm{a}}$ & 1.85 & 2.20 & 2.15 \\
\hline Density $\left[\mathrm{g} / \mathrm{cm}^{3}\right]$ & 8.445 & 8.28 & 7.13 \\
\hline Peak of emission & $1 / \lambda^{2}$ & $440 / 530$ & 480 \\
\hline Scintillation yield $[\mathrm{ph} / \mathrm{MeV}]$ & 0 & 200 & $8 \mathrm{k}-10 \mathrm{k}$ \\
\hline Low wavelength cutoff [nm] & 250 & 350 & 320 \\
\hline Slow/fast constant [ns] & $-/-$ & $30 / 6$ & $300 /-$ \\
\hline Yield ratio & - & 0.7 & - \\
\hline \multirow{2}{*}{ Crystal size $\left[\mathrm{mm}^{3}\right]$} & $(3 \times 3 \times 15)$ & $(3 \times 3 \times 15)$ & $(3 \times 3 \times 15)$ \\
\hline & $(3 \times 3 \times 20)$ & $(3 \times 3 \times 20)$ & $(3 \times 3 \times 20)$ \\
\hline
\end{tabular}

${ }^{\mathrm{a}}$ At the wavelength of peak emission.

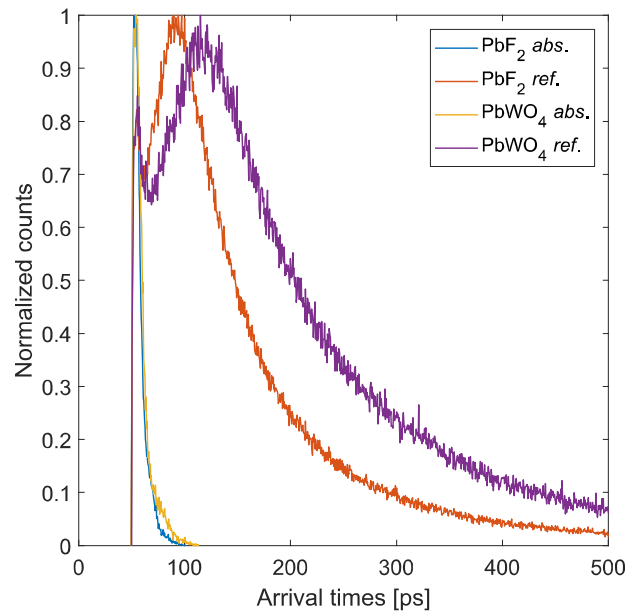

Fig. 2. Normalized PDFs of photon arrival times at the output surface of $\mathrm{PbF}_{2}$ and $\mathrm{PbWO}_{4}(3 \times 3 \times 15) \mathrm{mm}^{3}$. Abs. -absorbing surface (black painted); ref. reflective surface ( 0.98 reflectivity).
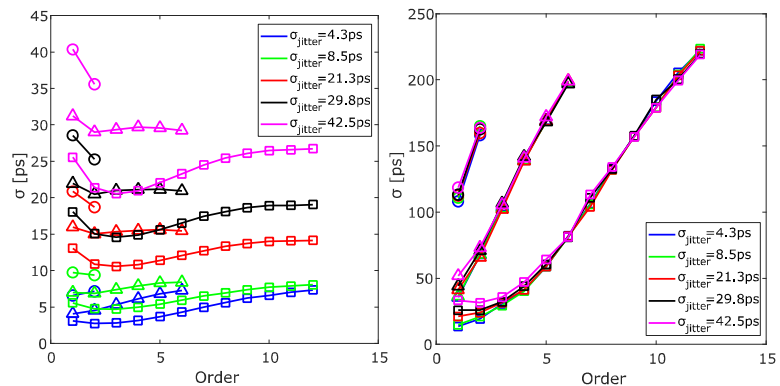

Fig. 3. Standard deviations of the arrival times for up to the first 12 detected photons, for three different average Cherenkov counts per frame $(2-$ circles, 6 - triangles, 12 - squares) and five jitter values, using $(3 \times 3 \times 15) \mathrm{mm}^{3} \mathrm{PbF}_{2}$ with black painted coating (left) and reflective coating (right).
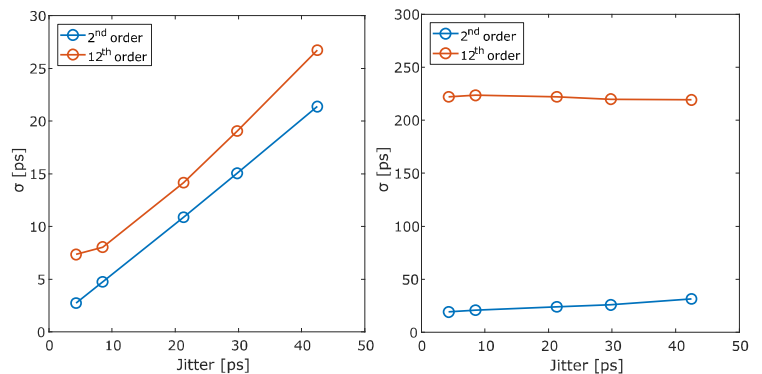

Fig. 4. Standard deviations of the arrival times for the $2^{\text {nd }}$ and $12^{\text {th }}$ detected photons, for an average of 12 Cherenkov counts per frame, as a function of jitter values, using $(3 \times 3 \times 15) \mathrm{mm}^{3} \mathrm{PbF}_{2}$ with black painted coating (left) and reflective coating (right).

\section{CONCLUSION}

Initial simulations have been carried out that study the effect of electronic jitter and radiator material on the timing resolution for Cherenkov detection. Additional effects, such as different coating materials and sizes, will be analyzed. DCR will be included in the mathematical model and the present simulations will be compared to the analytical solution.

\section{REFERENCES}

[1] P. Lecoq, M. Korzhik and A. Vasiliev, "Can Transient Phenomena Help Improving Time Resolution in Scintillators?," IEEE Transactions on Nuclear Science, vol. 61, pp. 229-234, 2014.

[2] W. Moses, "Time of flight in PET revisited," IEEE Transactions on Nuclear Science, vol. 50, pp. 1325 - 1330, 2003.

[3] S. Seifert, H. T. v. Dam and D. R. Schaart, "The lower bound on the timing resolution of scintillation detectors," Physics in Medicine and Biology, vol. 57, 2012.

[4] R. Dolenec, S.Korpar, P.Križan and R.Pestotnik, "Cherenkov TOF PET with silicon photomultipliers," Nuclear Instruments and Methods in Physics Research Section A: Accelerators, Spectrometers, Detectors and Associated Equipment, vol. 804, pp. 127-131, 2015.

[5] J. D. Thiessen et.al, "Performance evaluation of SensL SiPM arrays for high-resolution PET," chez Nuclear Science Symposium and Medical Imaging Conference, Seoul, 2013.

[6] J. Allison, et.al, "Geant4 developments and applications," IEEE Transactions on Nuclear Science, vol. 53, pp. 270 - 278, 2006.

[7] E. Venialgo, S. Mandai, T. Gong, D. R. Schaart and E. Charbon, "Time estimation with multichannel digital silicon photomultipliers," Physics in Medicine \& Biology, vol. 60, pp. 2435-2452, 2015. 